\title{
Essential postmitochondrial function of p53 uncovered in DNA damage-induced apoptosis in neurons
}

\author{
AE Vaughn ${ }^{1}$ and M Deshmukh ${ }^{*, 1,2}$
}

In postmitotic sympathetic neurons, unlike most mitotic cells, death by apoptosis requires not only the release of cytochrome $c$ from the mitochondria, but also an additional step to relieve X-linked inhibitor of apoptosis protein (XIAP)'s inhibition of caspases. Here, we examined the mechanism by which XIAP is inactivated following DNA damage and found that it is achieved by a mechanism completely different from that following apoptosis by nerve growth factor (NGF) deprivation. NGF deprivation relieves XIAP by selectively degrading it, whereas DNA damage overcomes XIAP via a p53-mediated induction of Apaf-1. Unlike wild-type neurons, p53-deficient neurons fail to overcome XIAP and remain resistant to cytochrome $c$ after DNA damage. Restoring Apaf-1 induction in p53-deficient neurons is sufficient to overcome XIAP and sensitize cells to cytochrome $c$. Although a role for p53 in apoptosis upstream of cytochrome $c$ release has been well established, this study uncovers an additional, essential role for p53 in regulating caspase activation downstream of mitochondria following DNA damage in neurons.

Cell Death and Differentiation (2007) 14, 973-981. doi:10.1038/sj.cdd.4402084; published online 12 January 2007

Apoptosis is a highly regulated genetic process that is crucial for the development of the entire organism, including the nervous system. Yet, apoptosis also plays an important role in neuronal injury and disease. Aberrant apoptosis can result in too little cell loss as seen in cancer progression, as well as too much cell loss such as following stroke, spinal cord injury and in many neurodegenerative diseases. ${ }^{1}$

A crucial event of apoptosis is the activation of caspase proteases. In mammalian cells, including neurons, caspase activation can be triggered by the release of cytochrome $c$ from the mitochondria. ${ }^{2,3}$ Once released, cytochrome $c$ binds to Apaf-1, inducing its oligomerization and subsequent recruitment of the initiator caspase, procaspase- 9 , to form the apoptosome complex. This complex results in activation of caspase-9, which can then cleave and activate downstream caspases such as caspase-3, which are responsible for cell death. $^{3}$

These major components of the apoptotic pathway have been well characterized through the use of cell-free biochemical studies and in intact cells using mitotic cell lines. However, recent evidence suggests that apoptosis is regulated very differently between mitotic and postmitotic cells. For example, in many mitotic cells such as HeLa cells, HEK 293 cells, primary fibroblasts and naïve PC12 cells, the addition of cytochrome $c$ to cytosolic extracts or injection of cytochrome $c$ into the cytosol of these cells induces rapid caspase activation and apoptosis. ${ }^{4-8}$ In contrast, cytochrome $c$, although necessary, is not sufficient to induce apoptosis in postmitotic cells such as sympathetic neurons, differentiated PC12 cells and cardiomyocytes. ${ }^{2,5,9,10}$ Recently, the X-linked inhibitor of apoptosis protein (XIAP) was identified as the critical regulator of caspase activation in these postmitotic cells. ${ }^{9,11}$ XIAP is a member of a family of inhibitor of apoptosis proteins (IAPs) that have been shown to regulate caspases by directly binding to and inhibiting their function. ${ }^{12}$ Whereas cytosolic microinjection of cytochrome $c$ is insufficient to induce apoptosis in wild-type neurons and cardiomyocytes, it is capable of doing so in XIAP-deficient neurons and cardiomyocytes. ${ }^{9,11}$

Although sympathetic neurons are resistant to injection of cytochrome $c$ into their cytosol, they are capable of undergoing cytochrome c-dependent apoptosis when induced by insults such as NGF deprivation and DNA damage. ${ }^{13-15}$ Therefore, in order to undergo apoptosis, neurons must activate signaling events that cause cytochrome $c$ release as well as inactivate XIAP. The mechanism by which neurons overcome XIAP's inhibition of caspases to become sensitive to cytochrome $c$ has been termed 'development of competence' and has been examined in the model of NGF deprivation. ${ }^{2}$ In NGF deprivation-induced death, the 'development of competence' is dependent neither on Bax function nor on protein synthesis, but occurs by the selective degradation of XIAP. ${ }^{11}$ Whether other apoptotic stimuli, most importantly those involved in pathological situations that engage the cytochrome $c$-mediated apoptosome pathway, use the same or different mechanisms to overcome XIAP remains unknown.

Previous studies have shown that DNA damage can activate the intrinsic apoptotic pathway leading to cytochrome

${ }^{1}$ Department of Cell and Developmental Biology, University of North Carolina, Chapel Hill, NC, USA and ${ }^{2}$ Neuroscience Center, University of North Carolina, Chapel Hill, NC, USA

*Corresponding author: M Deshmukh, Neuroscience Center 7109E Neuroscience Research Building, University of North Carolina, Box 7250, 105 Mason Farm Road, Chapel Hill, NC 27599, USA. Tel: 919843 6004; Fax: 919966 1050; E-mail: mohanish@med.unc.edu

Keywords: p53; apoptosis; cytochrome $C$; XIAP

Abbreviations: NGF, nerve growth factor; XIAP, X-linked inhibitor of apoptosis; $\mathrm{CHX}$, cycloheximide

Received 26.7.06; revised 30.10.06; accepted 30.10.06; Edited by DR Green; published online 12.1.07 
$c$ release and caspase-dependent cell death in many cell types including sympathetic neurons. ${ }^{13,16}$ Although DNAdamaging agents are commonly used as chemotherapeutics in the treatment of many cancers, they can also cause widespread neurotoxicity in patients. ${ }^{17}$ DNA damage has also been observed in neurodegenerative diseases. ${ }^{16,18}$ Therefore, understanding how apoptosis is regulated in neurons in response to DNA damage has clinical significance.

The tumor suppressor p53 has been implicated in the regulation of apoptosis in response to DNA damage in mitotic cells as well as neurons. ${ }^{16,19,20}$ p53 has been shown to regulate cytochrome $c$ release from the mitochondria both directly $^{21,22}$ and through the transcriptional induction of proapoptotic $\mathrm{Bcl}-2$ proteins such as Bax, Puma and Noxa. ${ }^{20}$ In this report, we find that p53 has an additional function to regulate apoptosis even after the point of mitochondrial cytochrome $c$ release in neurons.

We find that the mechanisms by which sympathetic neurons overcome XIAP's inhibition of caspases are distinct between NGF deprivation and DNA damage. Specifically, whereas NGF deprivation induces competence to relieve XIAP by targeting it for degradation, XIAP's inhibition of caspases after DNA damage is overcome by a p53-dependent increase in Apaf-1. Thus, in the absence of p53, DNA-damaged neurons are unable to execute apoptosis in response to cytochrome $c$ because they are incapable of relieving XIAP. These results identify an essential postmitochondrial role of p53 in regulating apoptosis in mammalian neurons.

\section{Results}

Etoposide induces cytochrome $c$ release and apoptosome-dependent death. Sympathetic neurons undergoing apoptosis mediated by the apoptosome pathway require not only the release of cytochrome $c$ from mitochondria but must also overcome XIAP's strict inhibition of caspases. Although the mechanism by which XIAP is relieved has been previously studied in the model of NGF deprivation-induced neuronal death, ${ }^{11}$ here we examined how XIAP is overcome in sympathetic neurons undergoing apoptosis with DNA damage.

First, we established that sympathetic neurons treated with DNA-damaging agents undergo a cytochrome $c$ and apoptosome-mediated cell death. Sympathetic neurons were treated with the topoisomerase II inhibitor etoposide and cell death was assessed at various time points. In response to etoposide, greater than $70 \%$ of sympathetic neurons undergo cell death by $48 \mathrm{~h}$. This death was apoptotic as it could be blocked by the pan-caspase inhibitor zVAD-fmk (Figure 1a) and dying
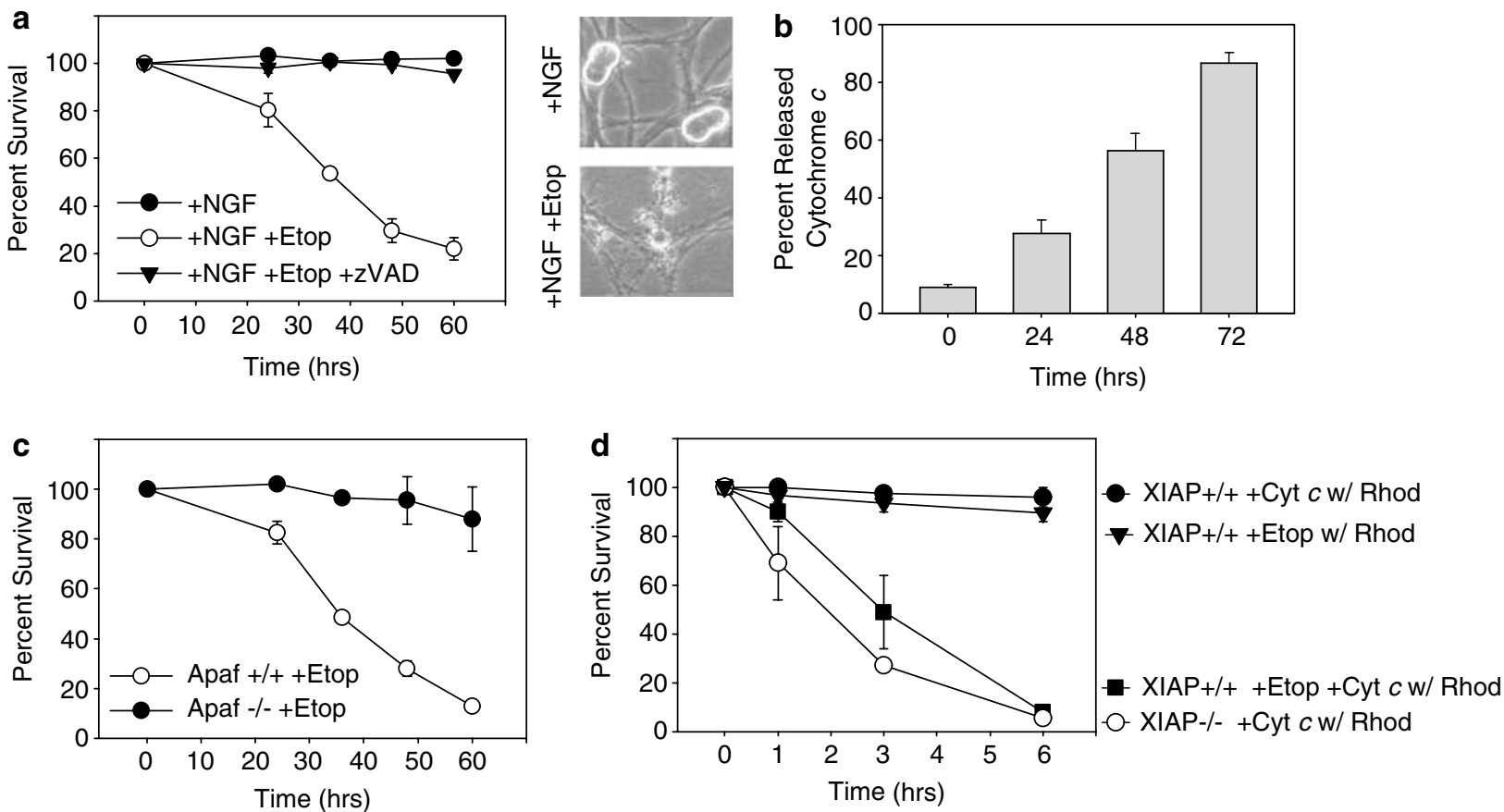

Figure 1 Etoposide induces cytochrome $c$ - and apoptosome-dependent apoptosis in sympathetic neurons. (a) Cultures of sympathetic neurons were either left untreated, treated with $20 \mu \mathrm{M}$ etoposide or treated with etoposide and the caspase inhibitor zVAD-fmk (50 $\mu \mathrm{M})$. At various time points, cell survival was assessed by morphological criteria (healthy neurons remain intact and phase bright). This method of assessing cell death correlates well with Trypan blue exclusion (Supplementary Figure S1A) and calcein AM staining. ${ }^{11}$ Data shown are mean \pm S.E.M. of three experiments. Photographs show untreated or etoposide-treated sympathetic neurons at $60 \mathrm{~h}$. (b) Cultures of sympathetic neurons were treated with $20 \mu \mathrm{M}$ etoposide for various time points and the status of cytochrome $c$ in these neurons was examined by immunocytochemical techniques. Neurons showing a punctate staining pattern have maintained cytochrome $c$ in the mitochondria, whereas neurons showing a loss of staining have released cytochrome $c$ from the mitochondria. Approximately 100 neurons per condition were counted to determine the status of cytochrome $c$. (c) Sympathetic neurons isolated from Apaf-1-deficient mice or their wild-type littermates were treated with $20 \mu \mathrm{M}$ etoposide and cell survival was assessed as described above. (d) Sympathetic neuronal cultures isolated from XIAP + I+ or XIAP-/- mice were injected with cytochrome $c$ and rhodamine dextran dye (Rhod) to mark injected cells, or rhodamine alone. Wild-type sympathetic neurons (XIAP $+/+)$ were also treated with etoposide $(20 \mu \mathrm{M})$ for $24 \mathrm{~h}$ or left untreated and then injected with cytochrome $c$ and rhodamine dextran dye (Rhod), or rhodamine alone. Cell survival of these neurons was then assessed at various time points following injection 
neurons exhibited Annexin V-positive staining (Supplementary Figure S1). Furthermore, this etoposide-induced death resulted in the release of cytochrome $c$ (Figure 1b) and engaged the apoptosome pathway. Unlike wild-type neurons, sympathetic neurons isolated from Apaf-1 deficient mice were resistant to etoposide (Figure 1c). Likewise, caspase-3 deficient sympathetic neurons have also recently shown to be resistant to etoposide-induced apoptosis. ${ }^{23}$

In sympathetic neurons undergoing apoptosis via the cytochrome $c$-mediated apoptosome pathway, the removal of XIAP's inhibition on caspases is necessary to permit death. Wild-type neurons are resistant, whereas XIAP-deficient neurons rapidly undergo apoptosis following injection of cytochrome $c$ (Figure 1d). ${ }^{11}$ The observation that etoposide engages death via the apoptosome pathway indicates that etoposide signaling must relieve XIAP's inhibition of caspases in order for cells to die. Indeed, etoposide treatment for $24 \mathrm{~h}$ (a time point at which cytochrome $c$ release and cell death are minimal) is able to relieve inhibition of caspases as it sensitizes wild-type neurons to cytochrome $c$ (Figure 1d). These results indicate that etoposide activates a mechanism that makes neurons permissive for cytochrome $c$-induced apoptosis before the neurons reach the point of cytochrome $c$ release.

Unlike NGF deprivation, etoposide-induced death requires translation to overcome XIAP. In the model of NGF deprivation-induced neuronal death, XIAP is inactivated by its selective degradation (Figure 2a). We were surprised to find, however, that neurons treated with etoposide underwent apoptosis yet maintained their XIAP levels (Figure $2 \mathrm{a}$ and $\mathrm{b}$ ). These results indicate that the mechanisms by which NGF deprivation and DNA damage overcome XIAP are distinct.

The development of competence can be directly determined by assessing the sensitivity of neurons to microinjection of cytochrome $c$. Only after neurons develop competence (overcome XIAP activity) do they become sensitive to

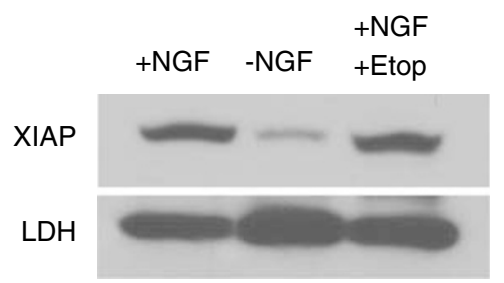

C

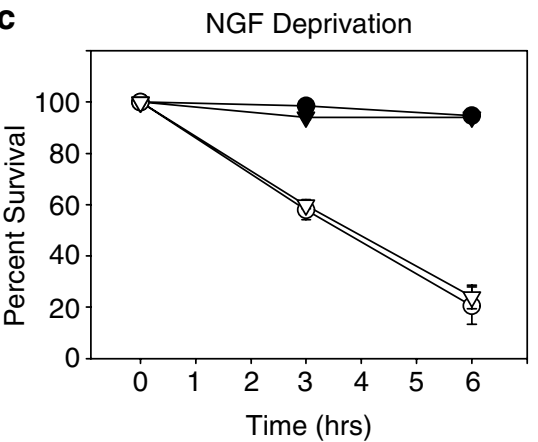

$$
\begin{aligned}
& \longrightarrow \text { Bax-/- -NGF +Rhod } \\
& - \text { - Bax-/- -NGF +Cyt } c \text { w/ Rhod } \\
& \longrightarrow-\text { CHX -NGF +Rhod } \\
& \rightarrow-\mathrm{CHX}-\mathrm{NGF}+\text { Cyt } c \text { w/ Rhod }
\end{aligned}
$$
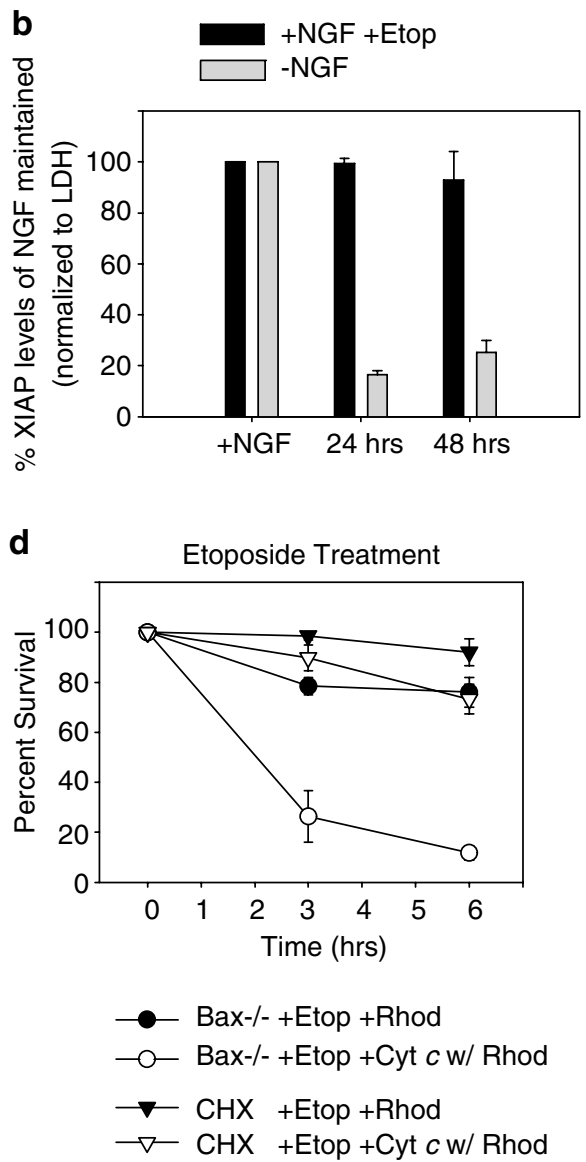

Figure 2 Development of competence by etoposide is independent of XIAP degradation and requires protein synthesis. (a) Protein levels of XIAP were examined from whole-cell lysates of sympathetic neurons maintained in NGF ( + NGF), deprived of NGF ( - NGF) for $24 \mathrm{~h}$ or treated with $20 \mu \mathrm{M}$ etoposide ( + NGF + Etop) for $24 \mathrm{~h}$. Levels of LDH were also examined as a loading control. (b) Quantitation of XIAP levels (mean \pm S.E.M. of three experiments) in NGF-deprived and etoposide-treated neurons at 24 and $48 \mathrm{~h}$ as compared to NGF-maintained neurons is shown and is normalized to LDH. (c) Cycloheximide (CHX) $(1 \mu \mathrm{g} / \mathrm{ml})$-treated wild-type sympathetic neurons or neurons isolated from Bax-deficient mice were deprived of NGF for $24 \mathrm{~h}$. Cells were then injected with $10 \mathrm{mg} / \mathrm{ml}$ cytochrome $c$ along with rhodamine dextran or with rhodamine dextran dye alone. At each time point after injections, the number of microinjected cells that remained viable was determined and expressed as a percentage of the total number of microinjected cells. (d) $\mathrm{CHX}(1 \mu \mathrm{g} / \mathrm{ml})$-treated wild-type sympathetic neurons or neurons isolated from Bax-deficient mice were treated with etoposide (20 $\mu \mathrm{M})$ for $24 \mathrm{~h}$. Cells were then injected with $10 \mathrm{mg} / \mathrm{ml}$ cytochrome $c$ along with rhodamine dextran or with rhodamine dextran dye alone. At each time point after injections, viable cells were quantified as in (c). Results shown are a mean ( \pm S.E.M.) of three experiments with 70-100 cells injected in each experiment 
cytosolic cytochrome $c$. Cytochrome $c$ release is dependent on Bax and protein synthesis, whereas in previous studies we have shown that neither Bax nor protein synthesis is required for the development of competence via degradation of XIAP after NGF deprivation. Therefore, NGF-deprived neurons, both Bax deficient and cycloheximide $(\mathrm{CHX})$ treated, are unable to release cytochrome $c$, yet develop competence and die with microinjected cytochrome $c$ (Figure $2 c)^{2}$

As the 'development of competence' pathway in etoposidetreated neurons appeared distinct and did not involve the degradation of XIAP, we asked whether this pathway was dependent on Bax or protein synthesis. Bax-deficient or wildtype cycloheximide-treated sympathetic neurons were exposed to etoposide for $24 \mathrm{~h}$, followed by microinjection of cytochrome $c$ and assessment of cell survival. Like the NGFdeprived condition, etoposide treatment induced competence in the Bax-deficient neurons (Figure 2d). However, unlike NGF-deprived neurons, etoposide-treated neurons failed to develop competence in the presence of cycloheximide and remained resistant to cytochrome $c$ (Figure $2 d$ ). These results indicate that the mechanism by which XIAP is overcome to develop competence in etoposide-treated neurons requires protein synthesis.

Apaf-1 is markedly induced in etoposide-treated sympathetic neurons. We have shown previously that Apaf-1 levels and the ability of XIAP to inhibit apoptosis are inversely coupled in cells. ${ }^{5}$ Under conditions where Apaf-1 levels are low, such as in postmitotic sympathetic neurons and cardiomyocytes, endogenous XIAP is an effective inhibitor of caspases. ${ }^{9,11}$ In contrast, in mitotic cells where Apaf- 1 levels are high, equivalent levels of XIAP are unable to strictly block caspase activation in response to cytosolic cytochrome $c^{5,9}$

Based on this observation, we speculated that an increase in Apaf-1 levels could be a mechanism by which sympathetic neurons disengage XIAP's brake on caspase activation in response to DNA damage. We examined whether Apaf-1 levels are increased in sympathetic neurons treated with etoposide. Indeed, Western blot analysis shows that Apaf-1 protein levels were elevated by $24 \mathrm{~h}$ following treatment with etoposide (Figure 3a). In contrast, no significant increase in Apaf-1 was seen after NGF deprivation. This increase in a

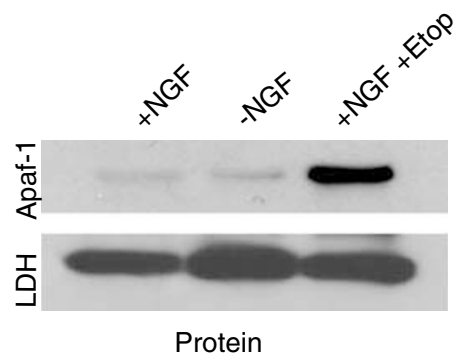

b

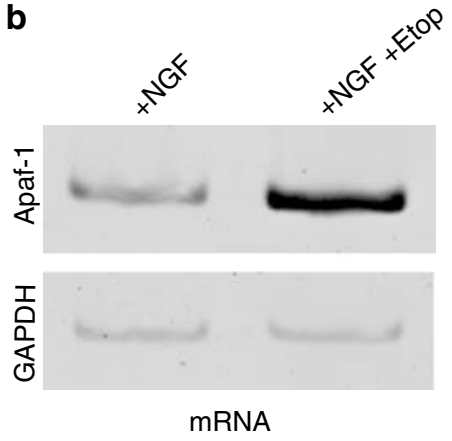

离
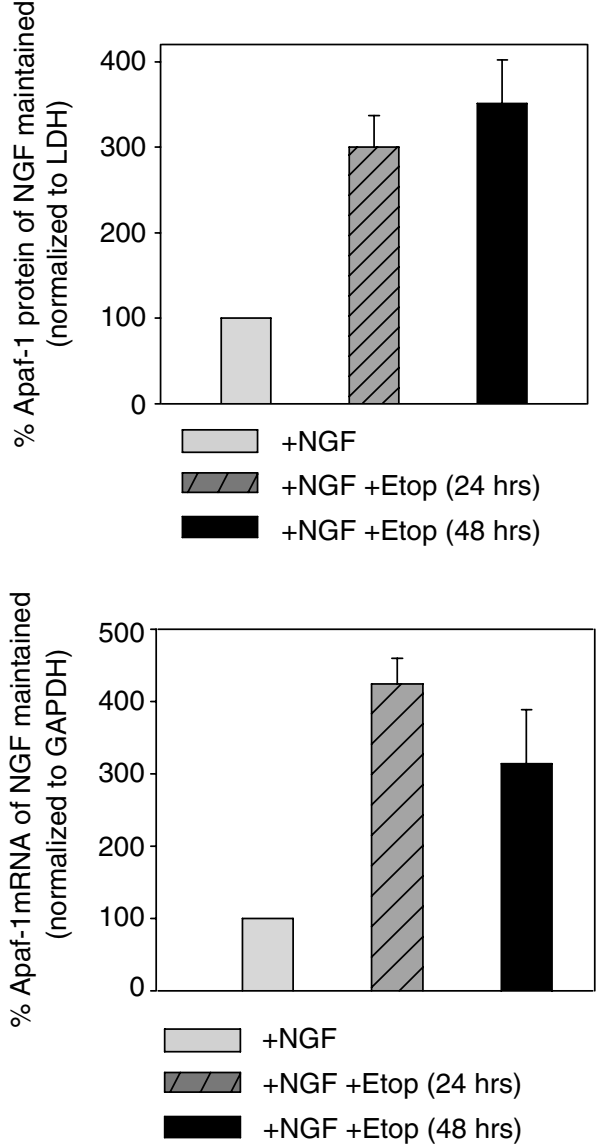

Figure 3 Etoposide treatment results in an increase in Apaf-1 protein and mRNA in sympathetic neurons. (a) Sympathetic neuronal cultures were either left untreated ( + NGF), deprived of NGF (-NGF) or treated with $20 \mu \mathrm{M}$ etoposide ( + NGF + Etop) for $24 \mathrm{~h}$. Cells were collected and levels of Apaf-1 protein analyzed by Western blot. $\mathrm{LDH}$ was used as a loading control. Quantitation of Apaf-1 protein levels normalized to LDH following etoposide treatment at 24 and $48 \mathrm{~h}$ is shown. Data are mean ( \pm S.E.M.) of three independent experiments. (b) Sympathetic neurons were either left untreated or treated with 20 etoposide for $24 \mathrm{~h}$. Levels of Apaf-1 and GAPDH (loading control) mRNAs were examined by quantitative RT-PCR and expressed as a percentage of NGF maintained as normalized to GAPDH. Quantitation shown is mean ( \pm S.E.M.) of three independent experiments 
Apaf-1 with DNA damage was also seen at the transcriptional level (Figure 3b). Similar increases in Apaf-1 have also been reported in cortical neurons following treatment with camptothecin or after traumatic brain injury. ${ }^{24,25}$ These data suggest that Apaf-1 induction could be necessary for disengaging XIAP's brake on caspase activation to permit apoptosis in neurons.

p53-deficient neurons are unable to develop competence in response to DNA damage. In the context of cell death, two functions of p53 have received much attention. First, p53 transcriptionally regulates proapoptotic Bcl-2 family members such as Bax, Puma and Noxa in response to DNA damage, which are important upstream activators of cytochrome $c$ release. ${ }^{26-28}$ Second, a transcriptional-independent role has been described for p53 in which it acts directly at the mitochondria to induce cytochrome $c$ release. ${ }^{21,22,29,30}$ Recently, p53 has also been identified as a transcriptional activator of Apaf-1, but the importance of this activity has remained unclear. ${ }^{24,31}$
As the increase in Apaf-1 could be a potential mechanism by which XIAP is relieved to permit neuronal apoptosis, we investigated the specific importance of p53 in this process. First, we examined whether p53 deficiency blocks apoptosis in sympathetic neurons in response to etoposide. While less than $10 \%$ of wild-type sympathetic neurons remained viable $60 \mathrm{~h}$ after etoposide treatment, greater than $80 \%$ of p53deficient neurons survived at this time (Figure 4a). As p53 has been shown to induce Puma, an event that is required for cytochrome $c$ release in response to DNA damage in sympathetic neurons, ${ }^{14}$ we anticipated that p53-deficient neurons might be resistant to apoptosis at multiple points in the apoptotic pathway. Consistent with this known premitochondrial function of p53 in neurons, while the majority of wildtype neurons had released cytochrome $c$ by $36 \mathrm{~h}$ of etoposide treatment, p53-deficient neurons retained cytochrome $c$ in the mitochondria (Supplementary Figure S2).

To specifically examine whether p53 is also important downstream of cytochrome $c$, we investigated whether p53-deficient neurons fail to become sensitive to cytochrome $c$ following DNA damage. Wild-type and p53-deficient
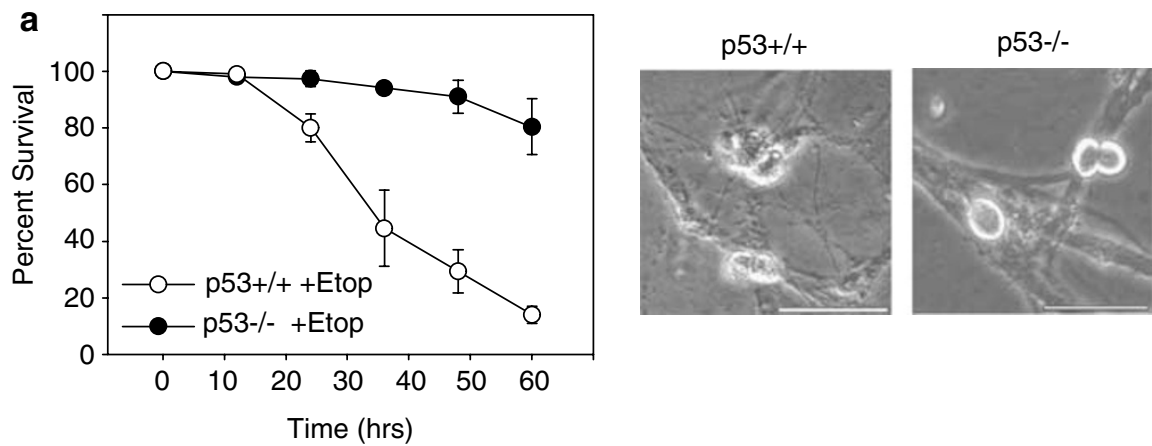

b

C
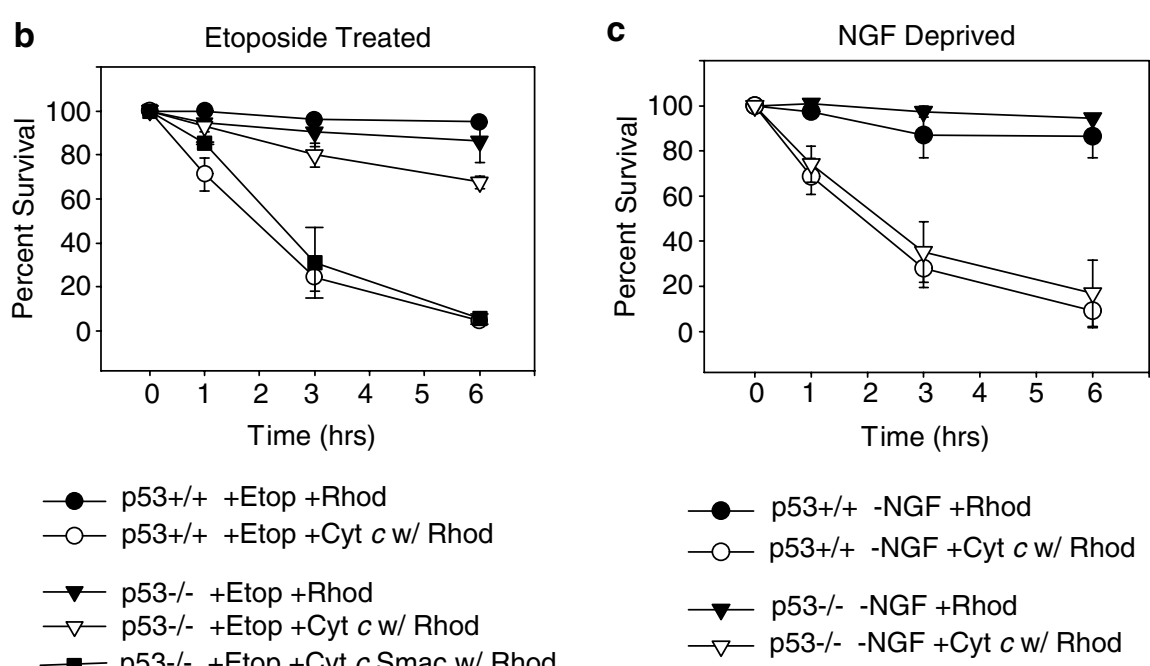

$$
\begin{aligned}
& \longrightarrow \text { p53+/+ +Etop +Rhod } \\
& \longrightarrow-\text { p53+/+ +Etop +Cyt } c \text { w/ Rhod } \\
& \rightarrow-\text { p53-/- +Etop +Rhod } \\
& \longrightarrow-\text { p53-/- +Etop +Cyt } c \text { w/ Rhod } \\
& \rightarrow-\text { p53-/- +Etop +Cyt } c \text { Smac w/ Rhod }
\end{aligned}
$$

Figure 4 p53-deficient neurons develop competence in response to NGF deprivation but not etoposide. (a) Cultures of sympathetic neurons from p53 + I + or p53-Imice were treated with $20 \mu \mathrm{M}$ etoposide. At various time points, cell survival was assessed by morphological criteria (healthy neurons remain intact and phase bright). Data are a mean \pm S.E.M. of three experiments with greater than 100 cells counted in each experiment. Representative photographs at $60 \mathrm{~h}$ after etoposide treatment are shown. Scale bars represent $50 \mu \mathrm{M}$. (b) p53 $+1+$ or p53-I- sympathetic neurons were treated with etoposide for $24 \mathrm{~h}$. Cells were then microinjected with cytochrome $c(10 \mathrm{mg} / \mathrm{ml})$ alone or cytochrome $c$ and Smac $(1 \mathrm{mg} / \mathrm{ml})$ together along with rhodamine dye, or rhodamine alone. Cell survival was assessed at various time points as indicated. (c) p53 $+/+$ or p53-/ - sympathetic neurons were deprived of NGF in the presence of cycloheximide $(1 \mu \mathrm{g} / \mathrm{ml})$ for $24 \mathrm{~h}$. Cells were then microinjected with cytochrome $c$ protein $(10 \mathrm{mg} / \mathrm{ml})$ along with rhodamine dye, or rhodamine alone. Cell survival was assessed at various time points as indicated 
neurons were treated with etoposide for $24 \mathrm{~h}$ (a time point at which the majority of neurons have not released cytochrome $c$; Supplementary Figure S2), followed by microinjection with cytochrome $c$. Activation of the apoptotic pathway directly at the point of cytosolic cytochrome $c$ is necessary as it allows one to bypass the known defect of cytochrome $c$ release found in p53-deficient neurons (Supplementary Figure S2). Unlike etoposide-treated wild-type neurons, which underwent apoptosis after cytochrome $c$ injections, etoposide-treated, p53deficient neurons remained resistant to cytochrome $c$ injection unless XIAP was removed by coinjection of Smac protein (Figure 4b). Injections with rhodamine dextran alone did not induce death in wild-type or p53-deficient etoposide-treated neurons.

To determine whether the inability of p53-deficient neurons to develop competence was specific to DNA damage, we asked whether p53-deficient neurons were able to develop competence in response to NGF deprivation. Wild-type or p53-deficient sympathetic neurons were deprived of NGF in the presence of cycloheximide (to prevent cytochrome $c$ release) for $24 \mathrm{~h}$. Consistent with our expectations, both wildtype and p53-deficient neurons developed competence and were sensitive to microinjection of cytochrome $c$ (Figure $4 c$ ). Thus, p53 is selectively important for the development of competence in response to DNA damage, which involves upregulation of Apaf-1, but not NGF deprivation, which involves the degradation of XIAP.

To confirm that the inability of p53-deficient neurons to undergo apoptosis in response to cytochrome $c$ was due to XIAP's inhibition of caspases, we asked whether p53-deficient neurons became sensitive to cytochrome $c$ following direct inactivation of XIAP. Indeed, injection of cytochrome $c$ along with Smac (an inhibitor of IAPs), but not cytochrome $c$ alone nor Smac alone, induced apoptosis in p53-deficient neurons, indicating that all the necessary apoptosomal components are present and capable of activation in the p53-deficient neurons if XIAP is removed (Supplementary Figure S3).

Thus, in sympathetic neurons, p53 is required to overcome XIAP (develop competence) to permit cytochrome $c$-mediated apoptosis following DNA damage.

\section{p53 links Apaf-1 induction with XIAP inactivation during} DNA damage-induced apoptosis in neurons. To investigate whether p53 is necessary for the induction of Apaf-1 protein in response to DNA damage, neurons from p53-heterozygous or p53-deficient mice were either left untreated or treated with etoposide. Western analysis revealed that whereas the levels of Apaf-1 protein increased following etoposide treatment in wild-type and p53-heterozygous neurons (Figures $3 a$ and $5 a$ ), Apaf- 1 was not induced in p53-deficient neurons under these conditions (Figure 5a). Thus, etoposide-mediated induction in Apaf-1 in sympathetic neurons was p53-dependent. We also note that p53 deficiency did not affect basal levels of Apaf-1, as Apaf- 1 levels were comparable between p53-heterozygous and p53-deficient neurons.

If the resistance of p53-deficient neurons to develop competence is due to the specific inability of these cells to upregulate Apaf- 1 in response to etoposide, then restoration of Apaf-1 induction in p53-deficient neurons would be expected to restore the ability of these neurons to develop competence following DNA damage. To test this, p53deficient sympathetic neurons were injected with plasmid DNA encoding either Apaf-1 or vector alone, along with EGFP to mark injected cells. After $24 \mathrm{~h}$, neurons were treated with etoposide for $24 \mathrm{~h}$, followed by microinjection of cytochrome $c$. Injection of cytochrome c into Apaf-1-expressing p53-deficient neurons resulted in complete apoptosis by $6 \mathrm{~h}$. However, a

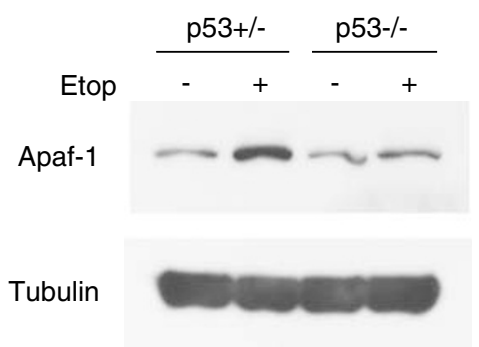

b

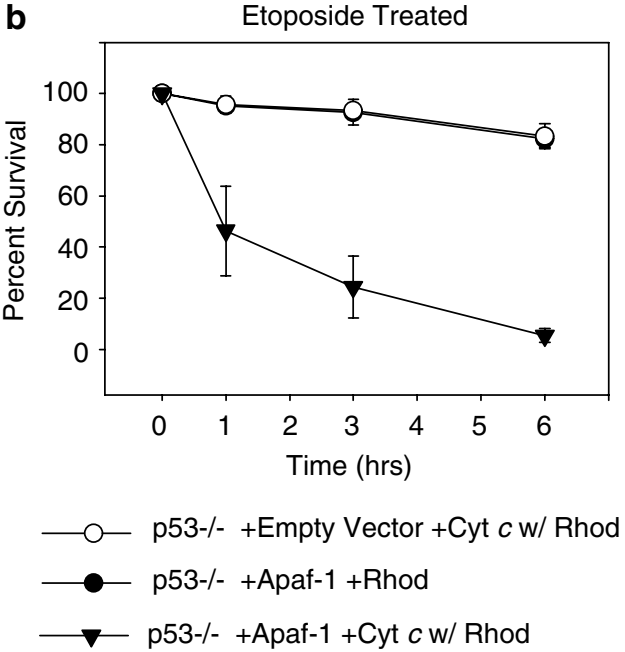

Figure 5 p53 links Apaf-1 induction with XIAP inactivation during DNA damage-induced neuronal apoptosis. (a) p53 $+/$ - or p53-/ - sympathetic neurons were either left untreated or treated with etoposide $(20 \mu \mathrm{M})$ for $24 \mathrm{~h}$. Cells were then collected and Apaf-1 protein levels were analyzed by Western blot. Tubulin is used as a loading control. The data are representative of multiple experiments. (b) p53-/- sympathetic neurons were injected with either a plasmid encoding Apaf-1 (200ng/ $\mu$ ) along with EGFP $(50 \mathrm{ng} / \mu \mathrm{l})$, or empty vector $(200 \mathrm{ng} / \mu \mathrm{l})$ along with EGFP $(50 \mathrm{ng} / \mu \mathrm{l})$ and allowed to express DNAs for $24 \mathrm{~h}$. Cells were then treated with $20 \mu \mathrm{M}$ etoposide for $24 \mathrm{~h}(\mathrm{a}$ time at which endogenous cytochrome $c$ is retained in the mitochondria; Supplementary Figure S2), after which GFP-expressing cells were injected with either cytochrome $c$ protein (10 mg/ $\mathrm{ml}$ ) along with rhodamine, or rhodamine alone. Cell survival of double injected cells was assessed by morphological criteria at the indicated time points. Data are a mean \pm S.E.M. of three independent experiments with 70-100 cells injected per experiment. Note that the data represented with open and filled circles are closely overlapping 
injection of rhodamine alone into Apaf-1-expressing cells or cytochrome $c$ into cells expressing empty vector did not induce death (Figure $5 \mathrm{~b}$ ). These data show that by restoring the induction of Apaf-1 by overexpression, DNA-damaged, p53-deficient sympathetic neurons regained the ability to develop competence and overcome XIAP's inhibition of caspases.

Together, these data show that a p53-mediated induction of Apaf- 1 is necessary to overcome XIAP to permit cytochrome $c$ to induce apoptosis in neurons in response to DNA damage.

\section{Discussion}

In this study, we have examined how the strict inhibition of caspases by endogenous XIAP is relieved when neurons undergo apoptosis following DNA damage. We find that the mechanisms by which XIAP is overcome in sympathetic neurons undergoing developmental apoptosis by NGF withdrawal and pathological apoptosis by DNA damage are distinct. Whereas NGF deprivation relieves XIAP by its selective degradation, ${ }^{11}$ XIAP levels remained unchanged after DNA damage (Figure $2 a$ and $b$ ). In contrast to NGF deprivation, the development of competence after DNA damage required protein synthesis. Consistent with this requirement, we found that Apaf-1 was markedly induced in neurons undergoing apoptosis with DNA damage (Figure 3a). Our results identify an important function of p53 in the development of competence, as p53-deficient neurons failed to induce Apaf-1 after DNA damage (Figure 5a). We examined the specific importance of Apaf-1 upregulation in relieving XIAP during DNA damage-induced apoptosis, and found that in the absence of an Apaf-1 induction, p53-deficient neurons failed to develop competence in response to DNA damage (Figure 4b). Importantly, restoring Apaf-1 induction by overexpression of Apaf-1 in p53-deficient neurons restored their ability to relieve XIAP, permitting cytochrome $c$ to induce apoptosis (Figure $5 \mathrm{~b}$ ).

These results validate our previous model, which proposed that the effectiveness of endogenous XIAP to inhibit apoptosis is coupled to Apaf- 1 activity in cells. Mitotic cells, which express high levels of Apaf-1, readily undergo apoptosis in response to cytochrome $c$, without the need to eliminate endogenous XIAP. In contrast, sympathetic neurons have low levels of Apaf-1, allowing endogenous XIAP to effectively inhibit cytochrome $c$-dependent caspase activation. ${ }^{5}$ As Apaf-1 is the limiting component of the apoptosome in these neurons, this model predicts that elevating levels of Apaf- 1 alone would be sufficient to increase the number of functional apoptosomes and overcome XIAP. In this study, we describe a pathological situation in which the coupling of Apaf-1 and XIAP becomes a critical regulator of caspase activation. In response to DNA damage, sympathetic neurons overcome XIAP and develop sensitivity to cytochrome $c$ by specifically upregulating Apaf-1. Induction of Apaf-1 was the only step required by etoposide to sensitize p53-deficient neurons to cytochrome c. p53-deficient cells in which Apaf-1 induction had been restored by overexpression underwent apoptosis in response to cytochrome $c$ even in the absence of etoposide treatment (Supplementary Figure S4).
Strict control of caspase activation by XIAP in neurons presumably functions as a safety brake that would prevent apoptosis from occurring in the event of accidental mitochondrial damage resulting in cytochrome $c$ release. However, in situations where death is inevitable, such as during development or after irreparable DNA damage, the efficient removal of this brake is important as it would permit apoptosis thereby avoiding the negative side effects of necrosis. Indeed, both NGF deprivation and DNA damage activate specific pathways that efficiently relieve XIAP at a time that precedes the release of cytochrome $c$ from mitochondria (Figure $1 \mathrm{~d}$ and $4 \mathrm{~b}$ ). ${ }^{11}$ Thus, apoptosis in XIAP-deficient neurons, although no longer needing the pathway to relieve XIAP, is still dependent on the release of cytochrome $c$ and therefore occurs with a time course that is indistinguishable from wild-type neurons (Supplementary Figure S5). ${ }^{11}$

Although the major components of the intrinsic pathway of apoptosis are conserved in both developmental (NGF deprivation) and pathological (DNA damage) apoptosis in sympathetic neurons, this study has identified unique differences in the way this pathway is regulated between these two models. Removal of NGF in these neurons selectively targets XIAP for degradation, whereas DNA damage overcomes XIAP by a p53-mediated induction of Apaf-1 (model in Figure 6a). We note that biochemical and overexpression studies have shown that mature Smac can also directly bind XIAP and inhibit its activity. ${ }^{32}$ However, endogenous Smac does not function to relieve XIAP following DNA damage in neurons, as both wild-type and Smacdeficient sympathetic neurons undergo apoptosis with a similar time course following etoposide treatment (Figure 6b). Thus, neurons appear to have evolved multiple Smac-independent pathways that are sufficient to relieve XIAP and permit cytochrome $c$-dependent apoptosis. Similar pathways may also be engaged in other postmitotic cells such as cardiomyocytes where relieving XIAP is necessary for apoptosis.

Apaf-1 has been shown to be induced in a p53-dependent manner in response to camptothecin in cortical neurons. ${ }^{24}$ However, whether the p53-mediated induction of Apaf-1 was necessary for neuronal apoptosis was not specifically examined. Indeed, p53 deficiency blocks apoptosis in response to DNA damage in many cells including neurons. ${ }^{16,33}$ However, studies investigating the mechanism of p53 action have primarily focused on events preceding cytochrome $c$ release. For example, proapoptotic members of the $\mathrm{Bcl}-2$ family proteins such as Bax, Noxa and Puma are transcriptional targets of p53, and their upregulation can promote cytochrome $c$ release in cells. ${ }^{14,20}$ In addition, recent studies show that p53 can directly induce cytochrome $c$ release by localizing to mitochondria. ${ }^{21,22,29,30}$ Our results now uncover an essential postmitochondrial function of p53 in apoptosis. Although p53-mediated induction of Apaf-1 is likely to occur in multiple cell types exposed to DNA damage, we expect that it is required only in cells where the inactivation of $\mathrm{XIAP}$ is necessary for cytochrome $c$-mediated apoptosis, such as neurons and cardiomyocytes. In addition, as overexpression of XIAP is seen in many cancers, ${ }^{34}$ our results predict that p53 activity could also overcome XIAP's inhibition of caspases by elevating Apaf-1 in such cancers. 

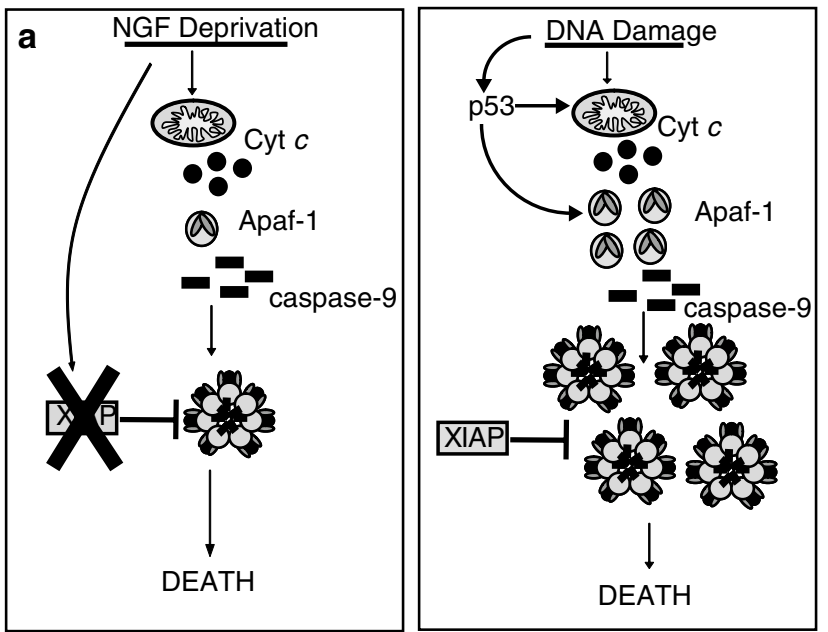

b

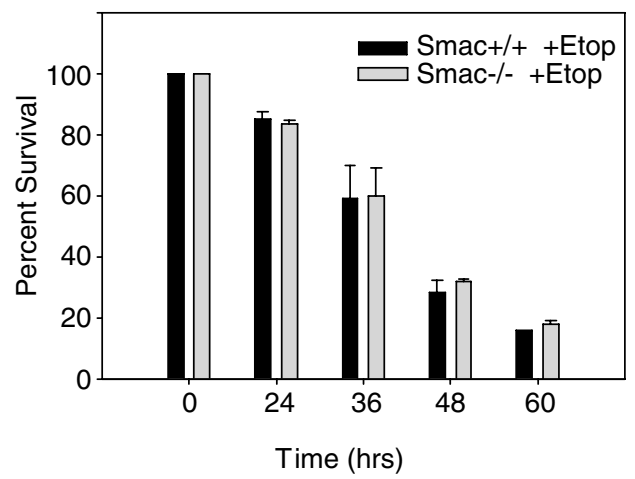

Figure 6 Distinct Smac-independent mechanisms by which XIAP is relieved in NGF deprivation and DNA damage models of neuronal apoptosis. (a) Although both NGF deprivation and DNA damage activate a pathway that releases cytochrome $c$ from the mitochondria, the mechanisms by which these two stimuli induce competence and overcome XIAP's inhibition of caspases are distinct. NGF deprivation selectively degrades XIAP, whereas DNA damage overcomes XIAP activity via a p53-mediated induction of Apaf-1. (b) Cultures of sympathetic neurons isolated from Smac-1- or wild-type mice were either left untreated or treated with $20 \mu \mathrm{M}$ etoposide. Quantitation of cell survival at various time points is shown

Several studies show that $\mathrm{p} 53$ is induced following a variety of neuronal injury paradigms such ischemia, hypoxia and excitotoxicity ${ }^{16,18}$ as well as in neurodegenerative diseases such as amyotrophic lateral sclerosis. ${ }^{35}$ Importantly, mice deficient in p53 are protected against glutamate or kainic acidexcitotoxic injury ${ }^{36}$ and ischemic brain damage. ${ }^{37}$ Our results predict that in these models, p53 deficiency would inhibit neuronal apoptosis not only by blocking cytochrome $c$ release but also by preventing the ability of these neurons to overcome XIAP, thus regulating apoptosis at both pre- and postmitochondrial steps.

Having multiple checkpoints in the regulation of apoptosis, and being able to precisely regulate these checkpoints, is critical for balancing a neuron's long-term survival with the need to undergo apoptosis when physiologically appropriate. An understanding of how these checkpoints are differentially regulated between mitotic and postmitotic cells is clinically significant as it allows for the development of therapeutics that could inhibit or activate apoptosis in selective cells in the context of neurodegenerative diseases or cancers.

\section{Materials and Methods}

Reagents. All reagents were purchased from Sigma (St Louis, MO, USA) or Fisher Scientific (Pittsburgh, PA, USA) unless otherwise stated. Collagenase and trypsin were purchased from Worthington Biochemical Corporation (Freehold, NJ, USA), and cycloheximide was purchased from Tocris Cookson (Ellisville, MO, USA). The pan-caspase inhibitor zVAD-fmk was purchased from Enzyme Systems Products. The Apaf-1 cDNA was a gift from Dr. Gabriel Nunez (University of Michigan, Ann Arbor, MI, USA). Recombinant Smac protein was purified from bacteria as described previously. ${ }^{11}$

Sympathetic neuronal cultures. Primary sympathetic neurons were dissected from the superior cervical ganglia of postnatal day 0-1 mice (P0-P1) and maintained in culture as described previously. ${ }^{38}$ Cells were plated on collagencoated dishes at a density of 60000 cells per well for Western or RT-PCR analysis, or 10000 cells per well for microinjection, survival counts or immunofluorescence experiments. Sympathetic neurons were grown for $4-5$ days in NGF-containing media before treating them with experimental conditions. For treatments, etoposide was used at a concentration of $20 \mu \mathrm{M}$. For NGF deprivation, cultures were rinsed three times with medium lacking NGF, followed by the addition of goat anti-NGF neutralizing antibody to this media. Other conditions required the addition of $1 \mu \mathrm{g} / \mathrm{ml}$ cycloheximide or $50 \mu \mathrm{M}$ zVAD-fmk.

ICR outbreed mice (Harlan Sprague Dawley) were used for all experiments except those involving Bax-, Apaf-1- and p53-deficient sympathetic neurons. The genetic background of Bax-, Apaf-1-, p53- and Smac-deficient mice is C57BL/6; wild-type littermates were used as controls in these experiments. Apaf-1-deficient mice were generated by Joachim Herz (UT Southwestern) ${ }^{39}$ and were kindly provided by the laboratory of Dr. Susan Ackerman (Jackson Laboratories). Heterozygous p53 mice were obtained from Jackson Laboratories (Maine). Smacdeficient mice were provided by the laboratory of Dr. Tak Mak (University of Toronto). Our methods for breeding and genotyping Bax-deficient mice have been described previously. ${ }^{40}$ The specific primers used to genotype Apaf-1-, p53- or Smac-deficient mice are listed in the online Supplementary materials and methods section.

Immunofluorescence analysis. The status of cytochrome $c$ (whether intact in the mitochondria or released) was examined by immunofluorescence. Briefly, sympathetic neurons were cultured for 4 days following which they were left untreated or treated with $20 \mu \mathrm{M}$ of etoposide for various time points. Neurons were then fixed in $4 \%$ paraformaldehyde and incubated overnight in anti-cytochrome $c$ primary antibody (556432, BD Biosciences) followed by a $2 \mathrm{~h}$ incubation with antimouse Cy3 secondary antibody (Jackson Labs). Nuclei were stained with Hoechst 33258 (Molecular Probes).

Microinjection and quantitation of cell survival. Our method of microinjecting sympathetic neurons with cytochrome $c$ has been described previously. ${ }^{5}$ Immediately after injections, the number of viable cells injected was determined by counting rhodamine-positive cells that had intact phase-bright bodies. This method of assessing neuronal survival correlates well with other cellsurvival assays such as Trypan blue exclusion and staining with calcein AM. ${ }^{11}$ At various times after injections, the number of viable injected neurons remaining was determined by using the same counting criteria and expressed as a percentage of the original number of microinjected cells.

In experiments involving microinjection of DNAs, the Apaf-1-expressing plasmid or empty vector was injected into neurons. The nucleus was injected with $200 \mathrm{ng} / \mu \mathrm{l}$ of the plasmid DNA along with $50 \mathrm{ng} / \mu \mathrm{l}$ of EGFP-expressing DNA (Clonetech laboratories Inc.) in microinjection buffer. After $24 \mathrm{~h}$ to allow for expression, GFPexpressing cells were identified by fluorescence microscopy and re-injected with cytochrome $c$. The survival of these double-injected cells was assessed by morphological criteria.

Western blot analysis. Western analysis was performed as described previously. ${ }^{11}$ The following antibodies were used; anti-Apaf-1 (13F11; Alexis Biochemicals), anti-XIAP (AF822; R\&D Systems), anti-LDH (Rockland), anti-alphatubulin (Sigma). Anti-mouse/rabbit HRP-conjugated secondary antibodies were purchased from Pierce Chemical Co. For quantitation, Western blots were developed with ECL-plus reagents (Amersham Biosciences) and analyzed on a Typhoon fluorescent imager (Amersham Biosciences) using Image Quant software (Amersham Biosciences). 
Quantitation of mRNA expression. Our method of quantitative RT-PCR analysis has been described previously. ${ }^{11}$ The gel was stained with Sybr Green (Molecular Probes) and visualized on a Typhoon fluorescence imager (Amersham Biosciences) and quantitated using ImageQuant software.

Annexin V and PI staining. Sympathetic neuronal cultures were treated with etoposide for $36 \mathrm{~h}$, followed by staining for Annexin $\mathrm{V}$ and propidium iodide (PI) using the TACS Annexin V-FITC Apoptosis Detection kit (R\&D Systems) according to the manufacturer's protocol. Briefly, cells were washed in PBS followed by a $15 \mathrm{~min}$ incubation with $2.5 \mu \mathrm{g} / \mathrm{ml} \mathrm{PI}$ and $1.25 \mu \mathrm{g} / \mathrm{ml}$ Annexin V-FITC. Cells were washed twice and viewed immediately on a Leica inverted fluorescence microscope.

Image acquisition and processing. All images were acquired by a Hamamatsu ORCA-ER digital B/W CCD camera mounted on a Leica inverted fluorescence microscope (DMIRE 2). The image acquisition software was Metamorph version 5.0 (Universal Imaging Corporation). Images were scaled down and cropped in Adobe Photoshop to prepare the final figures.

Acknowledgements. We thank Dr. Tak Mak (University of Toronto) for kindly providing the Smac-deficient mice. We also thank Kevin Wright, Michelle Smith and Yolanda Huang for critical review of this manuscript. This work was supported by NIH Grant NS42197.

1. Yuan J, Yankner BA. Apoptosis in the nervous system. Nature 2000; 407: 802-809.

2. Deshmukh M, Johnson Jr EM. Evidence of a novel event during neuronal death: development of competence-to-die in response to cytoplasmic cytochrome $c$. Neuron 1998; 21: 695-705.

3. Wang X. The expanding role of mitochondria in apoptosis. Genes Dev 2001; 15 2922-2933.

4. Liu X, Kim CN, Yang J, Jemmerson R, Wang X. Induction of apoptotic program in cell-free extracts: requirement for dATP and cytochrome c. Cell 1996; 86: 147-157.

5. Wright KM, Linhoff MW, Potts PR, Deshmukh M. Decreased apoptosome activity with neuronal differentiation sets the threshold for strict IAP regulation of apoptosis. J Cell Bio 2004; 167: 303-313

6. Li F, Srinivasan A, Wang Y, Armstrong RC, Tomaselli KJ, Fritz LC. Cell-specific induction of apoptosis by microinjection of cytochrome $c$. Bcl-XL has activity independent of cytochrome $c$ release. J Biol Chem 1997; 272: 30299-30305

7. Brustugun OT, Fladmark KE, Doskeland SO, Orrenius S, Zhivotovsky B. Apoptosis induced by microinjection of cytochrome $c$ is caspase-dependent and is inhibited by Bcl-2. Cell Death Differ 1998; 5: 660-668.

8. Juin P, Hueber AO, Littlewood T, Evan G. c-Myc-induced sensitization to apoptosis is mediated through cytochrome $c$ release. Genes Dev 1999; 13: 1367-1381.

9. Potts MB, Vaughn AE, McDonough H, Patterson C, Deshmukh M. Reduced Apaf-1 levels in cardiomyocytes engage strict regulation of apoptosis by endogenous XIAP. J Cell Biol 2005; 171: 925-930.

10. Vyas $S$, Juin $P$, Hancock D, Suzuki $Y$, Takahashi R, Triller $A$ et al. Differentiationdependent sensitivity to apoptogenic factors in PC12 cells. J Biol Chem 2004; 279 30983-30993

11. Potts PR, Singh S, Knezek M, Thompson CB, Deshmukh M. Critical function of endogenous $\mathrm{XIAP}$ in regulating caspase activation during sympathetic neuronal apoptosis. J Cell Biol 2003; 163: 789-799.

12. Salvesen GS, Duckett CS. IAP proteins: blocking the road to death's door. Nat Rev Mol Cell Biol 2002: 3: 401-410.

13. Besirli CG, Deckwerth TL, Crowder RJ, Freeman RS, Johnson Jr EM. Cytosine arabinoside rapidly activates Bax-dependent apoptosis and a delayed Bax-independent death pathway in sympathetic neurons. Cell Death Differ 2003; 10: 1045-1058.

14. Wyttenbach A, Tolkovsky AM. The BH3-only protein Puma is both necessary and sufficient for neuronal apoptosis induced by DNA damage in sympathetic neurons. I Neurochem 2006; 96: 1213-1226.
15. Deshmukh M, Johnson Jr EM. Programmed cell death in neurons: focus on the pathway of nerve growth factor deprivation-induced death of sympathetic neurons. Mol Pharm 1997; 51: 897-906.

16. Morrison RS, Kinoshita Y, Johnson MD, Guo W, Garden GA. p53-dependent cell death signaling in neurons. Neurochem Res 2003; 28: 15-27.

17. Lazarus HM, Herzig RH, Herzig GP, Phillips GL, Roessmann U, Fishman DJ. Central nervous system toxicity of high-dose systemic cytosine arabinoside. Cancer 1981; 48: 2577-2582.

18. Culmsee C, Mattson MP. p53 in neuronal apoptosis. Biochem Biophys Res Comm 2005; 331: 761-777.

19. Miller FD, Pozniak CD, Walsh GS. Neuronal life and death: an essential role for the p53 family. Cell Death Differ 2000; 7: 880-888.

20. Schuler M, Green DR. Mechanisms of p53-dependent apoptosis. Biochem Soc Trans 2001; 29: 684-688.

21. Marchenko ND, Zaika A, Moll UM. Death signal-induced localization of p53 protein to mitochondria. A potential role in apoptotic signaling. J Biol Chem 2000; 275: 16202-16212.

22. Chipuk JE, Kuwana T, Bouchier-Hayes L, Droin NM, Newmeyer DD, Schuler M et al. Direct activation of Bax by p53 mediates mitochondrial membrane permeabilization and apoptosis. Science 2004; 303: 1010-1014.

23. Wright KM, Vaughn AE, Deshmukh M. Apoptosome dependent caspase-3 activation pathway is non-redundant and necessary for apoptosis in sympathetic neurons. Cell Death Differ 2006; AOP, August 25, 2006; doi:10.1038/s..cdd.4402024.

24. Fortin A, Cregan SP, MacLaurin JG, Kushwaha N, Hickman ES, Thompson CS et al. APAF1 is a key transcriptional target for $\mathrm{p} 53$ in the regulation of neuronal cell death. $J$ Cell Biol 2001; 155: 207-216.

25. Yakovlev AG, Ota K, Wang G, Movsesyan V, Bao W-L, Yoshihara K et al. Differential expression of apoptotic protease-activating factor-1 and caspase-3 genes and susceptibility to apoptosis during brain development and after traumatic brain injury. J Neurosci 2001; 21: 7439-7446.

26. Oda E, Ohki R, Murasawa H, Nemoto J, Shibue T, Yamashita T et al. Noxa, a BH3-only member of the Bcl-2 family and candidate mediator of p53-induced apoptosis. Science 2000; 288: 1053-1058.

27. Yu J, Wang Z, Kinzler KW, Vogelstein B, Zhang L. PUMA mediates the apoptotic response to p53 in colorectal cancer cells. Proc Natl Acad Sci USA 2003; 100: 1931-1936.

28. Miyashita T, Reed JC. Tumor suppressor p53 is a direct transcriptional activator of the human bax gene. Cell 1995; 80: 293-299.

29. Chipuk JE, Bouchier-Hayes L, Kuwana T, Newmeyer DD, Green DR. PUMA couples the nuclear and cytoplasmic proapoptotic function of p53. Science 2005; 309: $1732-1735$

30. Mihara M, Erster S, Zaika A, Petrenko O, Chittenden T, Pancoska P et al. p53 has a direct apoptogenic role at the mitochondria. Mol Cell 2003; 11: 577-590.

31. Moroni MC, Hickman ES, Lazzerini Denchi E, Caprara G, Colli E, Cecconi F et al. Apaf-1 is a transcriptional target for E2F and p53. Nat Cell Biol 2001; 3: 552-558.

32. Verhagen AM, Vaux DL. Cell death regulation by the mammalian IAP antagonist Diablo/ Smac. Apoptosis 2002; 7: 163-166

33. Michalak E, Villunger A, Erlacher M, Strasser A. Death squads enlisted by the tumour suppressor p53. Biochem Biophys Res Commun 2005; 331: 786-798.

34. Liston $P$, Fong WG, Korneluk RG. The inhibitors of apoptosis: there is more to life than Bcl2. Oncogene 2003; 22: 8568-8580.

35. Martin LJ. p53 is abnormally elevated and active in the CNS of patients with amyotrophic lateral sclerosis. Neurobiol Dis 2000; 7: 613-622.

36. Morrison RS, Wenzel HJ, Kinoshita Y, Robbins CA, Donehower LA, Schwartzkroin PA Loss of the p53 tumor suppressor gene protects neurons from kainate-induced cell death. J Neurosci 1996; 16: 1337-1345.

37. Crumrine RC, Thomas AL, Morgan PF. Attenuation of p53 expression protects against focal ischemic damage in transgenic mice. J Cereb Blood Flow Metab 1994; 14: 887-891.

38. Deshmukh M, Vasilakos J, Deckwerth TL, Lampe PA, Shivers BD, Johnson Jr EM Genetic and metabolic status of NGF-deprived sympathetic neurons saved by an inhibitor of ICEfamily proteases. J Cell Biol 1996; 135: 1341-1354.

39. Honarpour N, Du C, Richardson JA, Hammer RE, Wang X, Herz J. Adult Apaf-1-deficient mice exhibit male infertility. Dev Biol 2000; 218: 248-258.

40. Deckwerth TL, Elliott JL, Knudson CM, Johnson Jr EM, Snider WD, Korsmeyer SJ. Bax is required for neuronal death after trophic factor deprivation and during development. Neuron 1996; 17: 401-411.

\section{Supplementary Information accompanies the paper on Cell Death and Differentiation website (http://www.nature.com/cdd)}

